\title{
3D pressure field reconstruction from time-resolved stereoscopic PIV measurements by relaxation of Taylor's hypothesis
}

\author{
D. Fratantonio ${ }^{1 *}$, J. J. Charonko ${ }^{1}$ \\ ${ }^{1}$ Los Alamos National Laboratory, Physics Division, Los Alamos, NM USA \\ *dfratantonio@lanl.gov
}

\begin{abstract}
This work presents reconstructions of 3D pressure fields starting from 2D3C stereoscopic-PIV (SPIV) measurements. In Fratantonio et al. (2021), we presented a new reconstruction algorithm, the "Instantaneous convection" method, capable of producing 3D velocity fields from time-resolved SPIV measurements. For reconstructions in flows with strong shear layers and high turbulence intensity, this method is able to provide time-resolved 3D velocity volumes that are more accurate than those that can be obtained from the more frequently employed reconstruction method based on the Taylor's hypothesis and on the use of a mean convective field. Here we investigate the possibility of reconstructing the $3 \mathrm{D}$ pressure field from the timeresolved series of reconstructed $3 \mathrm{D}$ velocity data. A pseudo-tracking method is employed for computing the velocity material derivative, and the pressure field is then reconstructed by solving the 3D Poisson equation. The velocity and pressure reconstructions are validated on the Direct Numerical Simulation data of the turbulent channel flow taken from the John Hopkins Turbulence Database (JHTDB), and an application to experimental SPIV measurements of an air jet flow in coflow carried out at the Turbulent Mixing Tunnel (TMT) facility at Los Alamos National Laboratory is presented.
\end{abstract}

\section{Introduction}

The last two decades have seen a fast development and improvement of tomographic PIV/PTV techniques (Elsinga et al., 2006; Novara and Scarano, 2013), and, with the increasing availability of time-resolved 3D3C velocity data, the existing algorithms for 2D pressure reconstruction (Charonko et al., 2010) have been extended to the 3D case and further improved to increase accuracy (Wang et al., 2016) and computational efficiency (Huhn et al., 2016). Although tomographic PIV/PTV are now established techniques for performing 3D velocity measurements in fluid flows, the required experimental setup is still considered complex and expensive, since it requires at least 4 cameras and high-repetition, high-power laser systems in timeresolved measurements. Therefore, any alternative methods capable of providing accurate time-resolved 3D velocity and pressure fields from less complex or expensive techniques are of great interest for the scientific community. In this perspective, several authors exploited the Taylor's hypothesis of frozen turbulence for converting time-correlation information of single-point or 2D measurements into missing spatial-correlation information. In applications of the Taylor's hypothesis to SPIV measurements (Ganapathisubramani et al., 2008), the time-resolved 2D3C velocity data $\boldsymbol{u}(x, y)$ at the SPIV measurement plane is converted into a 3D velocity volume by application of the following hyperbolic operator, $\frac{\partial u}{\partial t}+\bar{w}(x, y) \frac{\partial u}{\partial z}=\mathbf{0}$, with $\bar{w}(x, y)$ being the local mean out-of-plane velocity. We refer to this type of 3D velocity reconstruction as the "Mean convection" (MC) method.

It has been practically demonstrated, however, that the Taylor's hypothesis breaks down in highlyturbulent and/or shear flows (Lin, 1953; Dennis and Nickels, 2008; Zaman and Hussain, 1981). Large-scale turbulent structures cannot be considered "frozen" in a shear layer since they deform under the effect of the transverse spatial gradients of the mean velocity profile, and velocity fluctuations might convect the turbulent patterns with convective speed and direction different from those given by the local mean velocity. In this context, we recently proposed a new algorithm of 3D velocity reconstruction from SPIV data (Fratantonio et al., 2021), which we called the "Instantaneous convection" (IC) method and which we demonstrated 
providing large 3D velocity volumes in shear flows that are more accurate than those provided by the MC method. The IC method is a step-by-step Lagrangian-Eulerian reconstruction that (i) convects the 2D3C velocity data in the 3D space with the local instantaneous velocity vector $\boldsymbol{u}=(u, v, w)$ and that (ii) "unfreezes" the 3D flow through an iterative Poisson regularization of intermediate velocity reconstructions. It is the combining effect of these two new features in the reconstruction process that produces the key difference between the IC method and the MC method. Since the convective field used in the IC method for transporting the flow properties in the 3D space corresponds to the local instantaneous velocity, enforcing the divergence-free condition through the Poisson equation does not provide a simple regularization of the 3D field, but also introduces a temporal and spatial development of the convective field itself, thus recovering those non-linear fluid deformation mechanisms that would be otherwise lost when convecting the flow with a "frozen" mean velocity profile. A brief description of the IC algorithm is provided in Section 2.1.

Our next goal is to investigate the possibility of recovering $3 \mathrm{D}$ pressure fields from time-resolved series of 3D velocity fields reconstructed from SPIV measurements. The pressure field can be reconstructed by integration in the spatial domain of the pressure gradients. At present, there are two major classes of integration methods, i.e., direct line integration of the pressure gradients or Poisson-based pressure solver (Van Oudheusden, 2013). Both reconstruction strategies first require measurements of the material flow acceleration, $\frac{D u}{D t}$, which can be obtained with different approaches, depending on what type of velocity data is available (Van Gent et al., 2017). From time-resolved PTV measurements, the particle trajectories are directly measured and can locally provide $\frac{D u}{D t}$ data, which can then be interpolated on a Cartesian grid and integrated in space for pressure reconstruction by means of sophisticated processing tools, such as FlowFit (Gesemann et al., 2016) and VIC+ (Schneiders and Scarano, 2016). In this context, the recently proposed "Shake-theBox" method (Schanz et al., 2016) enabled applications of tomographic-PTV with particle seeding densities comparable to that of tomographic-PIV, thus providing accurate and dense material acceleration data able to enhance accuracy of pressure reconstruction. Our reconstructed 3D3C velocity data resemble more what can be obtained from time-resolved tomographic-PIV measurements, from which the material acceleration can be evaluated either with an Eulerian approach, where $\frac{D u}{D t}$ is computed in a stationary reference frame from the temporal and spatial velocity derivatives (Jakobsen et al., 1997), or with a pseudo-tracking method, where imaginary particles are distributed in the domain and their trajectories are computed by forward and backward integration in time (Liu and Katz, 2006; Pröbsting et al., 2013). Other approaches that can approximate $\frac{D \boldsymbol{u}}{D t}$ from a single 3D3C snapshot exist, such as the Taylor's hypothesis approach of Laskari et al. (2016) or the IVIC method proposed by Schneiders et al. (2016), but the IC or the MC methods can provide time-series of velocity volumes with the same temporal resolution used in carrying out SPIV measurements, and we can therefore rely on time-resolved 3D data for performing pressure reconstructions.

The application of these techniques to reconstructed SPIV velocity volumes has not been performed to date, not even for velocity volumes reconstructed by the common MC method. To the best of our knowledge, the work of de Kat and Ganapathisubramani (2012) is the only one that has tackled the problem of evaluating the accuracy of pressure fields reconstructed from SPIV velocity data in conjunction with the Taylor's hypothesis, but they limited the analysis to the reconstruction of $2 \mathrm{D}$ pressure fields by means of a 2D Poisson equation derived from the residual between the Taylor operator and the full Navier-Stokes equations. They did not perform reconstructions of 3D pressure fields directly from large 3D reconstructed velocity fields. In Section 3, both the IC and the MC method are validated by application to a Direct $\mathrm{Nu}$ merical Simulation (DNS) dataset of the turbulent channel flow taken from the John Hopkins Turbulence Database (JHTDB), and an error analysis in terms of reconstructed 3D velocity and pressure fields is presented. Section 4 demonstrates an application of the reconstruction algorithms to an experimental dataset of SPIV measurements of an air jet flow in co-flow performed in the Turbulent Mixing Tunnel (TMT) facility at Los Alamos National Laboratory.

\section{Reconstruction algorithms}

\subsection{Velocity reconstruction}

The way the IC method performs the reconstruction has direct effects on the resulting 3D velocity and, consequently, on the resulting 3D pressure field. Although all the details of the IC method can be found in Fratantonio et al. (2021), in this section we briefly describe the main structure of the algorithm, which will help in understanding the origins of eventual differences between the true fields and the reconstructed fields.

Starting from a set of SPIV measurements taken over a $x, y$-plane with a sampling time step $\Delta t_{s}$, the IC method processes the $2 \mathrm{D} 3 \mathrm{C}$ velocity planes through an iterative scheme. At each reconstruction iteration, 
the flow system is evolved forward/backward in time for a time step $\Delta t_{\text {rec }}=n \Delta t_{s}$, which is chosen as an integer multiple $n$ of the SPIV sampling time step, during which a subset $n$ of SPIV planes is introduced in the system and is processed by the following steps:

1. data convection: each data point of the $2 \mathrm{D} 3 \mathrm{C}$ velocity planes are convected in the $3 \mathrm{D}$ space by the local instantaneous velocity $\boldsymbol{u}(x, y)$;

2. 3D interpolation: the resulting velocity data scattered in the $3 \mathrm{D}$ space are interpolated on a rectangular, regular cartesian grid;

3. Poisson regularization: the Poisson equation for the velocity

$$
\nabla^{2} \boldsymbol{u}=-\nabla \times \boldsymbol{\omega}
$$

is applied on the intermediate 3D velocity field, thus recovering a divergence-free flow;

4. planes re-initialization: new $2 \mathrm{D} 3 \mathrm{C}$ velocity planes are re-defined from the interior of the regularized $3 \mathrm{D}$ velocity field.

At the next algorithm iteration, the re-initialized planes along with new ones from the SPIV database are processed by the same 4 steps above. The temporal evolution of the flow system happens during the convection step, where virtual fluid particles are associated to each node of the 2D3C velocity plane. Because the SPIV data do not provide any information on the flow structure in the out-of-plane direction, it is not possible to know how the particle trajectories vary in time and space as soon as the particles leave the SPIV measurement plane. Therefore, during the convection step of the IC method, the virtual particles are convected in the 3D space at a constant-in-time local velocity, thus forming linear trajectories. The hyperbolic operator that governs the velocity field of this phenomenon is $\frac{D u}{D t}=0$, which implicitly corresponds to enforce zero pressure-gradients,

$$
\nabla p=0,
$$

during the dynamical evolution of the flow. This does not necessarily mean that the pressure integration performed on the reconstructed 3D velocity volumes will provide a pressure field with zero fluctuations, since the right form of the Navier-Stokes equation is used for pressure reconstruction. However, as it will be shown in Section 3 , the approximation introduced during the convection step produces evident signatures on the velocity and pressure fields, and represents the most important source of errors introduced by the IC reconstruction. This reconstruction error is, however, limited by the prompt application of the Poisson equation on the intermediate $3 \mathrm{D}$ velocity field, a regularization that emulates the application of pressure gradients that exist in a divergence-free flow.

The MC algorithm consists, instead, in simply convecting the 2D3C velocity data forward and backward in space by means of the out-of-plane component of the mean velocity profile, $\bar{w}(x, y)$, and then interpolating the final velocity data on a regular 3D Cartesian grid. A comparison of the resulting hyperbolic operator, i.e., $\frac{\partial u}{\partial t}+\bar{w}(x, y) \frac{\partial u}{\partial z}=\mathbf{0}$, with the Navier-Stokes equations also reveal that the implicit pressure-gradient field applied to the flow during the mean flow convection is (see also Laskari et al. (2016)):

$$
\nabla p=-[\boldsymbol{u}-\bar{w}(x, y) \hat{z}] \cdot \nabla \boldsymbol{u}
$$

As for the IC method, the distortions introduced by the pressure field of Eq. 3 has direct effects on the accuracy of the reconstructed 3D velocity and pressure fields.

The final result of both reconstruction algorithms is a series of velocity volumes on a 3D Cartesian grid with a time separation equals to $\Delta t_{s}$, i.e., the sampling time step used for performing SPIV measurements.

\subsection{Pressure reconstruction}

The reconstruction of the instantaneous pressure field requires first the evaluation of the pressure gradients, which can be inferred from the temporal and spatial evolution of the 3D velocity field:

$$
\nabla p=-\rho \frac{D \boldsymbol{u}}{D t}=-\rho \frac{\partial \boldsymbol{u}}{\partial t}+\boldsymbol{u} \cdot \nabla \boldsymbol{u}
$$


In Eq. 4, the viscous term has been neglected since its contribution is relevant only in flow regions very close to the wall (Schneiders et al., 2016). Computations of the viscous term showed it was 2-3 orders of magnitude smaller than the material acceleration term, and including it in the evaluation of the pressure gradient did not provide any visible difference. The evaluation of the velocity material derivative from the time-series of velocity volumes is performed by means of the pseudo-Lagrangian approach, which has been shown to be more precise than the Eulerian approach when dealing with convective flows (Violato et al., 2011). At the initial time $t_{0}$, virtual particles are distributed at each node of the 3D velocity Cartesian grid. Each particle trajectory is then reconstructed by evaluation of the particle location at times $t_{ \pm i}=t_{0} \pm i \Delta t_{s}$ for $i=1,2, \ldots, M$, with $M$ the number of forward or backward time steps. The particle position at the new time $t_{i+1}=t_{i} \pm \Delta t_{s}$ is produced by using the predictor-corrector method reported in Yu et al. (2012), in which the predictor step provides a first estimate of the particle location,

$$
\boldsymbol{x}_{p}^{*}\left(t_{i \pm 1}\right)=\boldsymbol{x}_{p}\left(t_{i}\right) \pm \Delta t_{s} \boldsymbol{u}\left(\boldsymbol{x}_{p}\left(t_{i}\right), t_{i}\right),
$$

and the corrector step provides the final particle position,

$$
\boldsymbol{x}_{p}\left(t_{i \pm 1}\right)=\boldsymbol{x}_{p}\left(t_{i}\right) \pm \Delta t_{s} \frac{1}{2}\left[\boldsymbol{u}\left(\boldsymbol{x}_{p}\left(t_{i}\right), t_{i}\right)+\boldsymbol{u}\left(\boldsymbol{x}_{p}^{*}\left(t_{i \pm 1}\right), t_{i \pm 1}\right)\right] .
$$

The material acceleration is then computed with a least squares approach, as proposed by Pröbsting et al. (2013). The velocity $\boldsymbol{u}_{p}\left(t_{ \pm i}\right)$ of the particles at time $t_{ \pm i}$ along the reconstructed trajectory is calculated by spline interpolation of the 3D3C velocity data. The material acceleration could be estimated using finite differences by considering any possible time difference $\Delta t_{i}=t_{i}-t_{0}$ with respect to the starting time $t_{0}$ and the corresponding velocity variation $\Delta \boldsymbol{u}\left(\Delta t_{i}\right)=u_{j}\left(\boldsymbol{x}_{p}\left(t_{i}\right)\right)-u_{j}\left(\boldsymbol{x}_{p}\left(t_{0}\right)\right)$. This results in an over-determined algebraic linear system for each component $j$ of the material acceleration of the form

$$
\Delta t \frac{D u_{j}}{D t}=\Delta u_{j}
$$

with $\Delta \boldsymbol{t}$ a vector gathering all time differences, and $\Delta \boldsymbol{u}_{j}$ gathering all corresponding velocity differences for the $j$-component. The least squares solution of Eq. 7 finally provides an estimation of the material acceleration at each node of the original 3D Cartesian grid:

$$
\frac{D u_{j}}{D t}=\left(\Delta \boldsymbol{t}^{T} \Delta t\right) \Delta \boldsymbol{t}^{T} \Delta \boldsymbol{u}_{j}
$$

At the volume boundaries, the forward/backward advection can move the virtual particles outside of the domain, where velocity information is inaccessible. The pressure gradients computed at the boundaries are therefore cropped out, so to provide more accurate boundary conditions for the pressure integration step.

The omni-directional integration method introduced by Liu and Katz (2006) is capable of efficiently minimizing the influence of local errors in the material acceleration on the final reconstructed pressure field. The Poisson-based pressure integration is instead known to be more prone to error propagation, especially when pure Neumann conditions are used in large domain (Pan et al., 2016). Nevertheless, the extension of the original 2D path integration method to 3D domains makes the number of required integration paths so large that a computational parallelization is necessary to make this pressure integration strategy affordable in terms of computational time (Wang et al., 2019). We, therefore, opted for a pressure integration based on the simpler and more computationally efficient Poisson equation. By taking the divergence of the momentum equation, the Poisson equation for the pressure reads:

$$
\nabla^{2} p=-\nabla \cdot\left(\rho \frac{D \boldsymbol{u}}{D t}\right)
$$

which is completed by applying Neumann boundary conditions at each boundary face of the rectangular domain:

$$
\frac{\partial p}{\partial n}=-\rho \frac{D \boldsymbol{u}}{D t},
$$

with $n$ the spatial coordinate normal to the boundary. The Laplacian operator is discretized by second-order centered differences, and the Neumann boundary conditions are discretized by adding a ghost point out of the domain. The resulting algebraic linear system is then solved by spectral decomposition, in which 


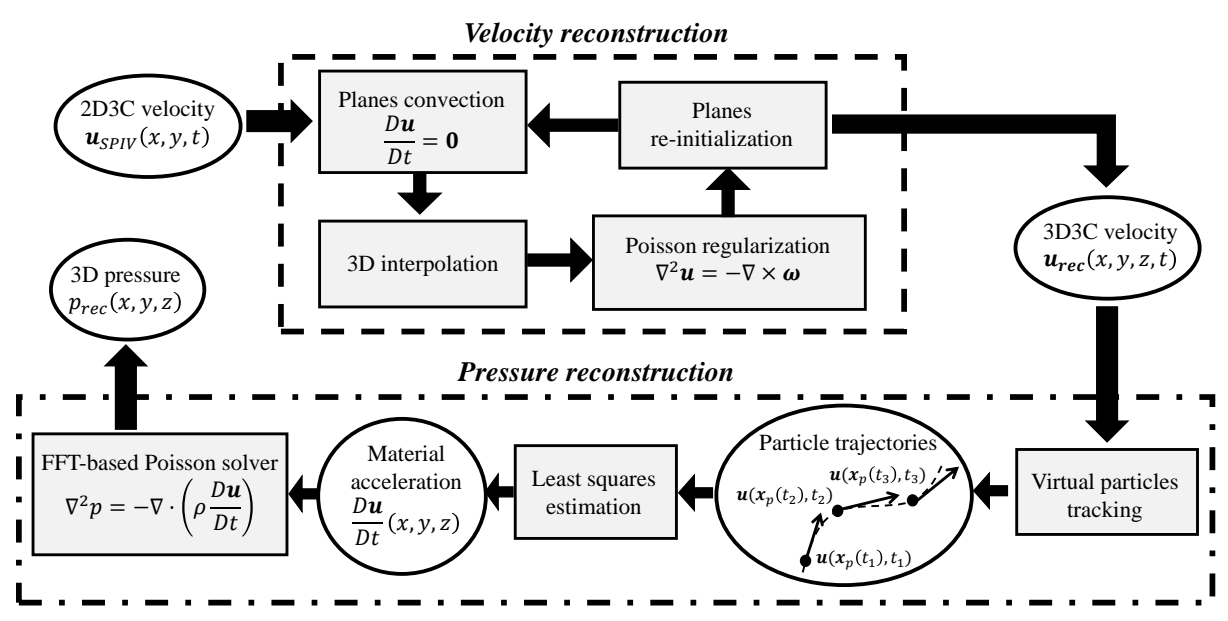

Figure 1: Schematic of the algorithm for 3D pressure reconstruction starting from SPIV velocity measurements.

the projection of the right hand side onto the matrix eigenspace is performed by means of the Fast Fourier Transform (FFT). In order to be able to employ the FFT for the projection, the eigenvector matrix has to be orthogonal and composed of cosine and sine functions. To mantain the eigenspace form as such while treating the Neumann boundary conditions with ghost points, the Poisson equation is solved on a staggered grid instead of the original Cartesian mesh. Finally, the resulting 3D pressure field is re-interpolated on the original grid, and the spatially averaged pressure over the entire volume is set to zero.

The resulting overall procedure for reconstructing 3D pressure fields from SPIV data is sketched in Figure1.

\section{Numerical validation}

The velocity and pressure 3D reconstructions provided by the IC and the MC methods are validated on the DNS data of the turbulent channel flow available on the JHTDB (Kim et al., 1987). In the following, all variables are non-dimensional, with the half-height channel $H$ being the reference length and the bulk velocity $W_{b}$ being the reference velocity. The Cartesian coordinate system is defined so that the $z$-axis is oriented towards the main flow direction, the $y$-axis represents the wall normal coordinate, and the $x$-axis is oriented in the spanwise direction of the channel. Synthetic SPIV 2D3C velocity data are sampled on a $x, y$-plane perpendicular to the main flow direction in a flow region close to the wall, i.e., in the range $150<y^{+}<250$, which is at the outskirt of the log-law region (Pope, 2001).

Since the JHTDB provides the DNS data on an inertial frame of reference moving in the flow direction at speed of 0.45 , the synthetic SPIV velocity measurements are also taken on the same moving reference frame. This does not pose any particular problem for the purpose of validating the reconstruction algorithms. It reduces the separation between the Lagrangian time scale, $\tau_{\eta}$, and the Eulerian time scale, which is defined by the convected Kolmogorov length scale as $\tau_{E u l}=\eta / \bar{W}$, with $\bar{W}$ the average speed of the flow in the region under consideration. For a given SPIV sampling time step $\Delta t_{s}$, this provides in turns a better resolution of the Eulerian spectrum and, thus, a higher spatial resolution of the reconstruction in the flow direction (see Fratantonio et al. (2021) for a more in-depth discussion of these aspects). The synthetic SPIV measurements are performed at a non-dimensional sampling time step of $\Delta t_{s}=0.0065$, thus resolving the smallest eddy turn-over times but under-resolving the highest frequency of Eulerian spectrum, which is what commonly happens in real SPIV measurements. Table 1 summarizes the most important flow characteristics, including the Kolmogorov time and length scales, estimated from the largest turbulent dissipation rate $\epsilon$ computed from the 3D DNS velocity, and the ratio of the SPIV sampling time step to the characteristic flow time scales. By defining the velocity fluctuations as $\boldsymbol{u}^{\prime}=\boldsymbol{u}-\overline{\boldsymbol{u}}$, the turbulence intensity is computed from

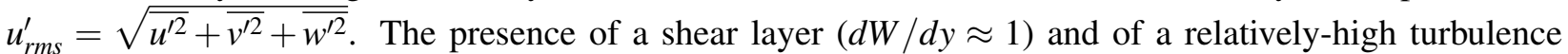
intensity $\left(u_{r m s}^{\prime} / \bar{W} \approx 20 \%\right.$ ) enhances the improvements provided by the IC method over the MC method, the latter being expected to fail in accurately reconstructing large-scale structures. 


\begin{tabular}{ccccccccc}
\hline$R e_{b}=2 H W_{b} / \nu$ & $\bar{W}$ & $\frac{u_{r m s}^{\prime}}{\bar{W}}$ & $\frac{d W}{d y}$ & $\eta=\left(\frac{v^{3}}{\varepsilon}\right)^{1 / 4}$ & $\tau_{\eta}=\left(\frac{v}{\varepsilon}\right)^{1 / 2}$ & $\tau_{\eta}=\frac{\eta}{W}$ & $\frac{\Delta t_{s}}{\tau_{\eta}}$ & $\frac{\Delta t_{s}}{\tau_{\text {Eul }}}$ \\
40000 & 0.49 & 0.2 & 1.16 & 0.0014 & 0.042 & 0.0026 & 0.155 & 2.5 \\
\hline
\end{tabular}

Table 1: Non-dimensional flow characteristics. The non-dimensional kinematic viscosity is $v=5 \cdot 10^{-5}$.

\subsection{Velocity and pressure reconstructions from synthetic SPIV data}

For the velocity reconstruction, each volume is generated from 500 synthetic SPIV planes. For reducing the computational time of each reconstruction, the IC algorithm processes $n=10$ planes at each iteration, thus the flow reconstruction evolves forward/backward in time with time steps of $\Delta t_{\text {rec }}=n \Delta t_{s}=0.065$. The reconstruction starts from $z=0$, the SPIV measurement location, and extends downstream, in the $z>0$ direction, i.e., the direction of the channel flow, and upstream, in the $z<0$ direction. For the pressure reconstruction, the virtual particle tracking is performed with $M=3$, by advancing the particles position 3 time steps forward and 3 time steps backward, for which 7 velocity volumes have been used.

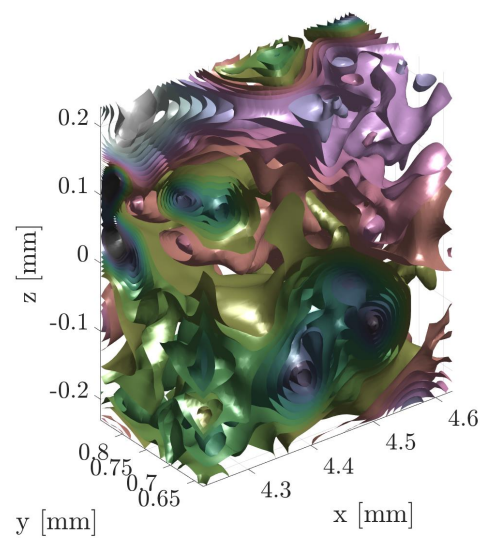

(a)

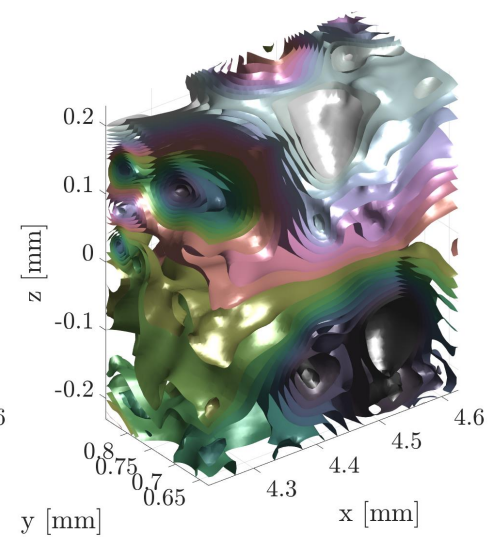

(b)

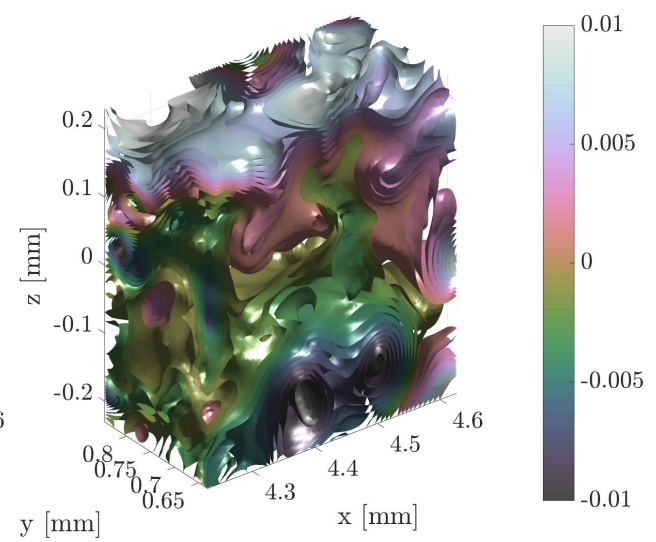

(c)

Figure 2: 3D pressure fields reconstructed from: (a) DNS velocity; (b) reconstructed IC velocity; (c) reconstructed MC velocity. The spatially averaged pressure is set to zero for all volumes.

The reconstructed 3D velocity fields are not shown here, but very similar results in terms of reconstructed $3 \mathrm{D}$ vorticity fields can be found in Fratantonio et al. (2021). Figure 2 reports isosurfaces of the reconstructed $3 \mathrm{D}$ pressure fields. The 3D pressure field in Figure 2 is reconstructed from the exact DNS velocity volumes. The DNS pressure field directly available from the JHTDB is in very good match with that of Figure 2 , thus validating the pressure reconstruction tools (the material derivative computation and the Poisson solver) developed for this work. Figures $2 \mathrm{p}$ and $2 \mathrm{k}$ show the pressure fields reconstructed by, respectively, the IC method and the MC method. In general, neither of the reconstruction methods are able to fully reproduce the pressure field correctly, especially on top and bottom of the volume, which are flow regions that are the furthest from the measurement plane location, $z=0$, where the reconstruction errors on the reconstructed velocity are the highest ones. The IC method seems providing a reconstructed pressure field that does not recover all small scale pressure fluctuations, but well reproduces large structures such as the two large negative pressure lobes in the upstream region, $-0.2<z<0$. In contrast, the pressure field reconstructed by the MC method show the same large structures in the wrong location, being convected with the wrong speed and distorted by the mean shear layer profile $\frac{\partial \bar{w}}{\partial y}$.

However, the 3D pressure fields shown in Figure 2 allow us to visualize only the results at the domain boundaries, where the reconstruction errors are the largest both for the velocity and pressure reconstructions. For a better and more meaningful comparison of the IC and MC reconstructions, we reported in Figure 3 the 2D maps of the vorticity and of the pressure fields at a $x$, z-plane cutting the 3D volumes of Figure 2 at the center, at about $y=0.73$. The vector fields on top of the 2D pressure maps correspond to the inplane velocity fluctuations $\left(u^{\prime}, w^{\prime}\right)$. A qualitative comparison of the 2D maps of Figure 3 demonstrate that the IC method provides better reconstruction than the MC method, both in terms of vorticity and pressure fields. With reference to Figure $3 \mathrm{k}$, the MC method tends to distort the flow field. The mean velocity 


\begin{tabular}{ccccc}
\hline & $u^{\prime}$ & $w^{\prime}$ & $\|\boldsymbol{\omega}\|$ & $p$ \\
IC & 0.973 & 0.996 & 0.887 & 0.898 \\
MC & 0.626 & 0.965 & 0.565 & 0.871 \\
\hline
\end{tabular}

Table 2: Correlation coefficients computed on the 2D maps of Figure 3 for different flow properties.

profile $\bar{w}(x, y)$ moves the vortical structures with the wrong speed, so that they end in the wrong position and stretched by the mean shear layer. For instance, by considering the two strong vortices in the upstream region $(-0.2<z<-0.1)$ indicated by the arrows in Figure 3, one of them is stretched in the flow direction, while the second one is not even present in the flow field, being convected too far upstream. The IC vorticity is instead in much better match with the exact vorticity field, with all vortical structures located in the right spot, and no distortion is present. The higher reconstruction accuracy of the IC method is further corroborated by the correlation coefficients reported in Table 2, which have been computed over the same 2D plane for different flow properties. It is, however, evident that the IC method tends to reduce the intensity of the strongest vortices in the field, as it happens for the intense vorticity regions indicated by the arrows in Figure 3. As also discussed in Fratantonio et al. (2021), these features are a direct consequence of how the 2D3C velocity data are convected in the 3D space. With the implicit zero-pressure gradients enforced on the flow during the convection step of the IC method, there is no pressure gradient field that would keep a vortex together as it happens in divergence-free flow. There are no pressure forces that would prevent those fluid portions at the vortex center that rotate faster to crash on and to compress outer fluid layers that rotate slower. This results in an expansion of the vortices, with a consequent weakening of their intensity. In the reconstructed pressure field, the amplitude of the negative pressure fluctuations at the center of the weakened vortices is, consequently, smaller.
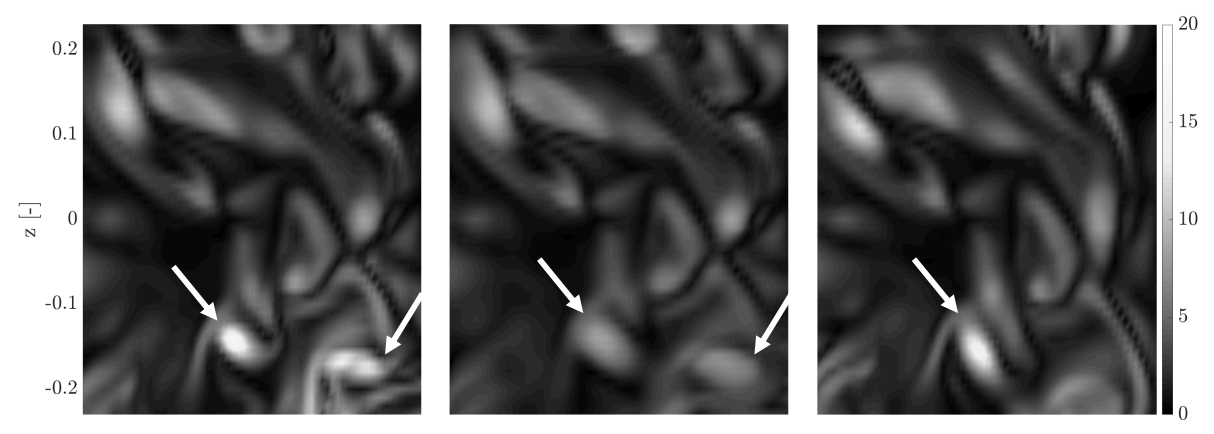

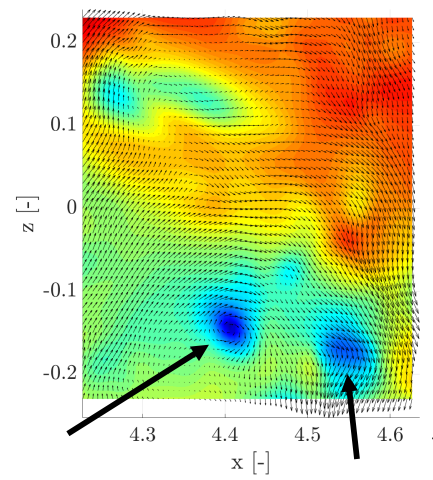

(a)

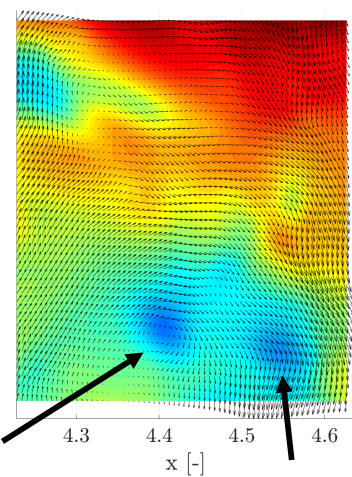

(b)

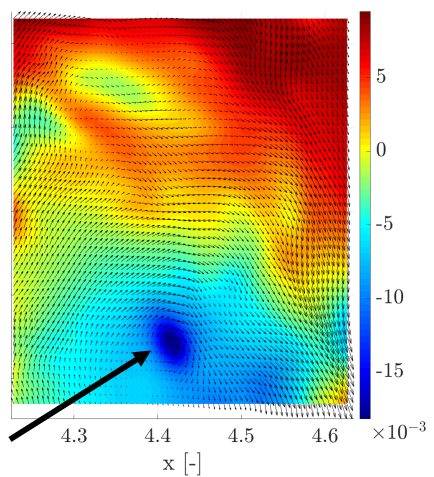

(c)

Figure 3: Instantaneous enstrophy (top row) and pressure (bottom row) fields over a 2D plane cutting the volumes of Figure 2 at $y=0.73$ : (a) DNS data; (b) IC reconstruction; (c) MC reconstruction. The 2D pressure map in (a) is extracted from the 3D pressure field reconstructed from the DNS velocity volumes. The vector fields on top of the pressure maps represent the in-plane velocity fluctuations $\left(u^{\prime}, w^{\prime}\right)$. The arrows indicate the same vortices and the corresponding negative pressure fluctuations regions across the various reconstructions. 


\subsection{Reconstruction error analysis}

For a more quantitative analysis of the reconstruction accuracy, we analyzed the error between the exact DNS fields and the reconstructed ones. For the velocity reconstruction, we define the relative error

$$
\varepsilon_{u}=\frac{\left\|\boldsymbol{u}_{r e c}-\boldsymbol{u}_{e x}\right\|_{2}}{\left\|\boldsymbol{u}_{e x}\right\|_{2}} .
$$

In Figure $4 \mathrm{a}$, we reported the average value of $\varepsilon_{u}$ computed over $x, y$-planes cutting the reconstructed 3D velocity field at different $z$-positions along the flow direction. In Figure $4 \mathrm{p}$, we also reported the correlation coefficient between the DNS vorticity and the reconstructed vorticity fields computed on the same 2D cuts as a function of $z$. As expected, the relative error $\varepsilon_{u}$ increases as a function of the distance from the SPIV measurement plane location, i.e., $z=0$, where the error is identically zero, since the reconstructed volume is centered on the synthetic SPIV velocity plane extracted directly from the DNS data. While the MC method provides $3 \mathrm{D}$ velocity fields with reconstruction errors that go up to more than $15 \%$, the IC method is able to provide reconstructions with about half the errors, with maximum values of less than $10 \%$. The improvements provided by the IC method are even more evident by looking at the results of Figure $4 \mathrm{~b}$, showing an evident drastic drop in the correlation between the DNS vorticity and the MC vorticity fields, even for reconstructed volumes as small as $z=[-0.05 ; 0.05]$, and a much better match of the IC reconstruction with the exact vorticity field, with correlation cofficients of about 0.9 even for reconstructed volumes as large as $z=[-0.15 ; 0.15]$. Although not shown here, curves very similar to those of Figure $4 \mathrm{~b}$ can be obtained for the turbulent dissipation rate $\epsilon$.

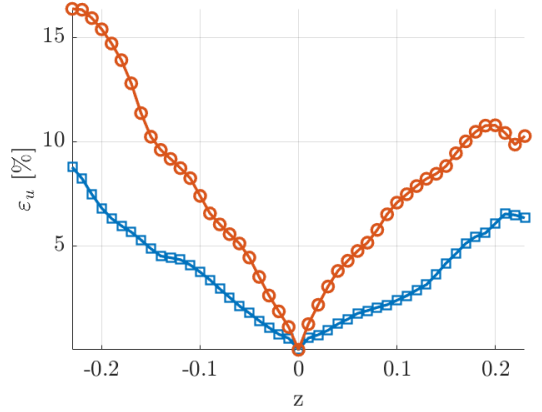

(a)

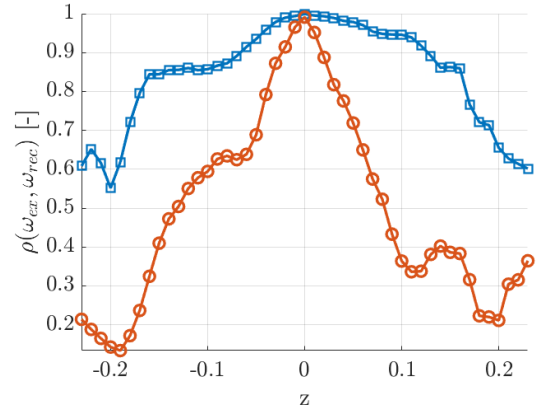

(b)

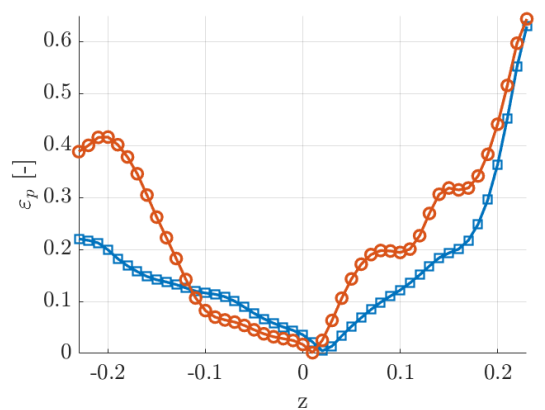

(c)

Figure 4: (a) Average velocity reconstruction error $\varepsilon_{u}$ as a function of $z$; (b) correlation coefficient between exact enstrophy field and reconstructed enstrophy field as a function of $z$; (c) absolute value of the average pressure reconstruction error $\varepsilon_{p}$ as a function of $z$. The square, blue symbols refer to the IC reconstruction, and the circle, brown symbols to the $\mathrm{MC}$ reconstruction. The average errors and the correlation coefficient are computed over $x, y$-planes at each $z$-location, with $z=0$ corresponding to the position of the SPIV measurement plane.

Defining a relative error for the pressure reconstruction similar to that of Eq. 11 gives error values that are very high, as high as $1000 \%$ in regions of the flow where the pressure value is close to zero. Nevertheless, the errors in the reconstructed 3D velocity fields are in the range 5-15\%, and, for such error levels in the velocity data, similar high relative errors on the reconstructed pressure has been observed even in application of the Poisson-based integration method to 2D flows (Charonko et al. 2010). For the same amount of errors in the velocity data, it has been observed that the omni-directional integration method is more efficient to average out the local errors in the pressure gradients computed from noisy velocity data, although the relative errors on the reconstructed pressure can still be very high in flow regions where the pressure is close to zero. For having a more meaningful comparison between the IC and the MC methods in terms of pressure reconstruction accuracy, we consider instead a definition of the pressure error similar to that proposed in Wang et al. (2019), in which the pressure error difference is normalized by the turbulent kinetic energy:

$$
\varepsilon_{p}=\frac{p_{r e c}-p_{e x}}{T K E_{e x}} .
$$


In Eq. 12, we defined $T K E_{e x}=\frac{1}{2} \rho\left(\overline{u_{e x}^{\prime 2}}+\overline{v_{e x}^{\prime 2}}+\overline{w_{e x}^{\prime 2}}\right)$ as a spatially-averaged turbulent kinetic energy of the DNS velocity fluctuations, and we considered as reference pressure $p_{e x}$ the $3 \mathrm{D}$ pressure field reconstructed from the DNS velocity volumes. Figure 4 c reports the average error $\varepsilon_{p}$ computed as a function of $z$. Since the errors in the velocity reconstructed by the MC method are higher, the IC algorithm performs in general better even in terms of pressure reconstructions, except in the region $-0.1<z<0$, where the IC errors are slightly higher than the MC ones. As for $\varepsilon_{u}$, the average error $\varepsilon_{p}$ is almost zero close to $z=0$ and increases with the distance from the SPIV measurement plane location, which indicates how the accuracy of the pressure reconstruction is directly affected by the local errors in the velocity data and the computed material acceleration.

An analysis of the local error distribution can provide a better insight on the exact origin of the errors in the velocity and pressure reconstructions. With reference to the 2D maps of Figure 3 , the local value of the pressure error $\varepsilon_{p}$ is shown in Figure 5. where the vector field represents the error on the reconstructed in-plane velocity fluctuations. The average value of the error distributions of Figure 5 are 0.004 and 0.0194 respectively for the IC and the MC pressure reconstructions. With reference to Figure 5, the local pressure error given by the MC reconstruction is almost everywhere higher than that given by the IC method. More precisely, the largest velocity and pressure errors in the IC reconstruction are localized at the center of the strongest vortices in the flow. As discussed in the previous section, the inherent expansion of the strong vortices that occurs during the convection step of the IC method results in weakening the intensity of the vortices and, consequently, in a lower magnitude of the depressions at the center of the same vortices.

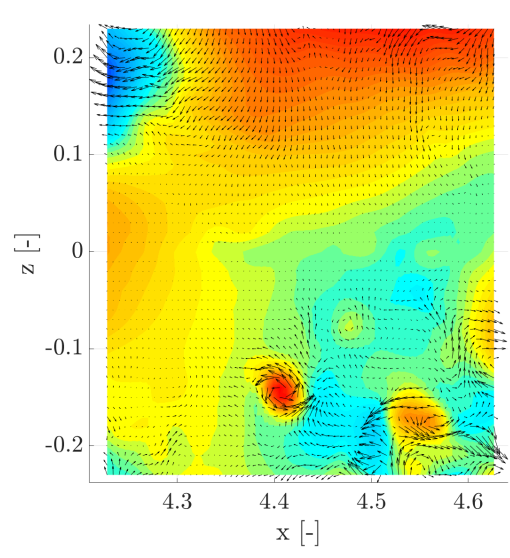

(a)

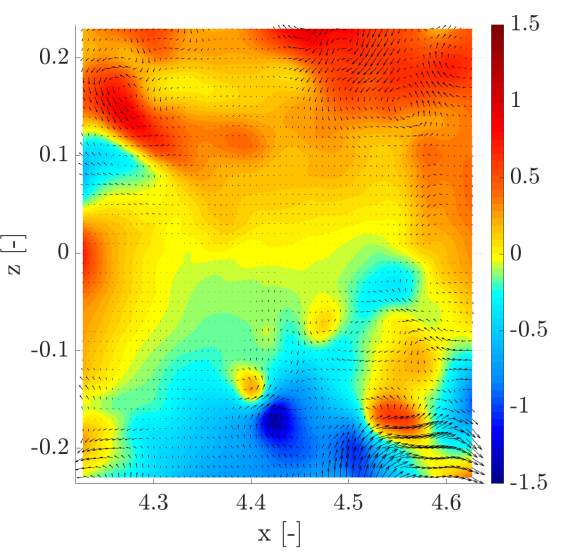

(b)

Figure 5: Local pressure reconstruction error $\varepsilon_{p}$ given by (a) the IC method and (b) the MC method for the $2 \mathrm{D}$ pressure field shown in Figure 3 . The vector field represents the error on the in-plane velocity fluctuations, i.e., $\left(u_{r e c}^{\prime}-u_{e x}^{\prime}, w_{r e c}^{\prime}-w_{e x}^{\prime}\right)$.

\section{Velocity-pressure reconstruction from SPIV measurements}

The reconstruction algorithms are applied to real experimental SPIV measurements performed in the Turbulent Mixing Tunnel facility at Los Alamos National Laboratory. The tunnel has a cross section area of $0.524 \times 0.524 \mathrm{~m}^{2}$, inside which an air jet is issued downward from a circular copper pipe with inner diameter $D=11 \mathrm{~mm}$, and a coflow is generated by a fan located downstream. SPIV measurements are carried out at a sampling frequency of $f_{s}=2 \mathrm{kHz}$ by using two high-speed Phantom ${ }^{\circledR}$ VEO 640S cameras working in dual-exposure mode, and a dual head Nd:YAG laser delivering $20 \mathrm{~mJ} /$ pulse at $532 \mathrm{~nm}$. The SPIV measurement plane is perpendicular to the jet flow direction and is located at a distance $16 \mathrm{D}$ downstream from the pipe outlet. Currently, the experimental facility does not include simultaneous, alternative diagnostic techniques for providing measurements of pressure or velocity, and, therefore, we cannot directly assess the accuracy of the velocity-pressure reconstructions. We limit the following analysis to a comparison of the reconstructions provided by the IC and the MC methods. For this experimental test, we considered an air jet with an average speed of $6.3 \mathrm{~m} / \mathrm{s}$ at the pipe outlet, which corresponds to a Reynolds number $R e=4250$, and a coflow speed of about $0.8 \mathrm{~m} / \mathrm{s}$. At the measurement location, the average speed at the jet centerline is $2.2 \mathrm{~m} / \mathrm{s}$. Figures $6 \mathrm{a}$ and $6 \mathrm{p}$ show the $3 \mathrm{D}$ vorticity and pressure fields reconstructed by, respectively, the 
IC method and the MC method. The appearance of more vortical structures in the MC reconstruction is partly due to the vortex-expansion issue affecting the IC convection and partly due to the smoothing effect of the iterative Poisson regularization. The vorticity and pressure reconstructions around the SPIV measurement plane are very much alike for the IC and MC methods, corroborating the error analysis of Section 3.2 . However, as the reconstruction develops in the upstream and downstream directions, the two reconstructions become qualitatively different in terms of position, orientation, and magnitude of the pressure and velocity fields, for which the numerical analysis of the previous section suggests that the IC reconstruction is more accurate than the MC one.

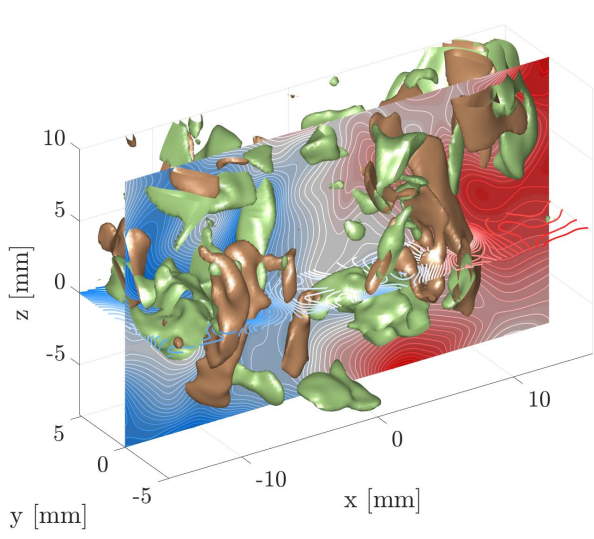

(a)

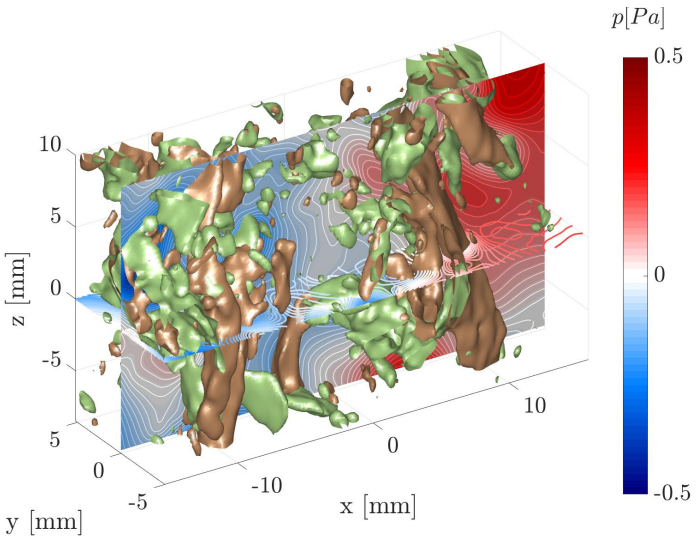

(b)

Figure 6: Reconstructed 3D velocity and pressure fields from experimental SPIV data: (a) IC reconstruction; (b) MC reconstruction. The brown isosurfaces correspond to the $z$-component of the vorticity with value $\left|\omega_{z}\right|=400 s^{-1}$. The green isosurfaces correspond to the azimuthal component of the vorticity with value

$\left|\omega_{\theta}\right|=500 s^{-1}$. The countour maps and lines correspond to the reconstructed pressure field.

\section{Conclusions}

In this work, we numerically and experimentally investigated 3D pressure reconstructions starting from 2D3C SPIV velocity measurements. We numerically demonstrated that the IC method introduced in Fratantonio et al. (2021) is more accurate than the MC method in reconstructing 3D velocity and pressure fields in turbulent flows with shear layer. In Section 4, we presented a practical demonstration of the reconstruction algorithms on real SPIV measurements of an air jet in coflow. As for the MC method, the IC method can be applied only to convective flows, in which the flow is crossing the SPIV measurement plane in one direction only. This guarantees the existence of a main flux of flow information filling the reconstruction domain. For this reason, the presence of a coflow around the main jet is required for extending the volumetric reconstruction to outer flow regions. The error analysis presented in Section 3.2 also revealed that the largest reconstruction errors introduced by IC method are mainly localized in the regions of strong vorticity. Currently, we are working in improving the IC algorithm from this point of view, thus further improving both 3D velocity and pressure reconstructions. The IC algorithm is, therefore, a promising processing tool for obtaining accurate 3D velocity and pressure fields from time-resolved SPIV measurements.

\section{References}

Charonko JJ, King CV, Smith BL, and Vlachos PP (2010) Assessment of pressure field calculations from particle image velocimetry measurements. Measurement Science and technology 21:105401

de Kat R and Ganapathisubramani B (2012) Pressure from particle image velocimetry for convective flows: a taylor's hypothesis approach. Measurement Science and Technology 24:024002

Dennis DJ and Nickels TB (2008) On the limitations of taylor's hypothesis in constructing long structures in a turbulent boundary layer. Journal of Fluid Mechanics 614:197 
Elsinga GE, Scarano F, Wieneke B, and van Oudheusden BW (2006) Tomographic particle image velocimetry. Experiments in fluids 41:933-947

Fratantonio D, Lai CC, Charonko J, and Prestridge K (2021) Beyond taylor's hypothesis: a novel volumetric reconstruction of velocity and density fields for variable-density and shear flows. Experiments in Fluids 62:1-25

Ganapathisubramani B, Lakshminarasimhan K, and Clemens N (2008) Investigation of three-dimensional structure of fine scales in a turbulent jet by using cinematographic stereoscopic particle image velocimetry. Journal of fluid mechanics 598:141

Gesemann S, Huhn F, Schanz D, and Schröder A (2016) From noisy particle tracks to velocity, acceleration and pressure fields using b-splines and penalties. in 18th international symposium on applications of laser and imaging techniques to fluid mechanics, Lisbon, Portugal. pages 4-7

Huhn F, Schanz D, Gesemann S, and Schröder A (2016) Fft integration of instantaneous 3d pressure gradient fields measured by lagrangian particle tracking in turbulent flows. Experiments in Fluids 57:1-11

Jakobsen M, Dewhirst T, and Greated C (1997) Particle image velocimetry for predictions of acceleration fields and force within fluid flows. Measurement Science and Technology 8:1502

Kim J, Moin P, and Moser R (1987) Turbulence statistics in fully developed channel flow at low reynolds number. Journal of fluid mechanics 177:133-166

Laskari A, de Kat R, and Ganapathisubramani B (2016) Full-field pressure from snapshot and time-resolved volumetric piv. Experiments in Fluids 57:44

Lin CC (1953) On taylor's hypothesis and the acceleration terms in the navier-stokes equation. Quarterly of Applied Mathematics 10:295-306

Liu X and Katz J (2006) Instantaneous pressure and material acceleration measurements using a fourexposure piv system. Experiments in fluids 41:227-240

Novara M and Scarano F (2013) A particle-tracking approach for accurate material derivative measurements with tomographic piv. Experiments in fluids 54:1-12

Pan Z, Whitehead J, Thomson S, and Truscott T (2016) Error propagation dynamics of piv-based pressure field calculations: how well does the pressure poisson solver perform inherently?. Measurement Science and Technology 27:084012

Pope SB (2001) Turbulent flows. IOP Publishing

Pröbsting S, Scarano F, Bernardini M, and Pirozzoli S (2013) On the estimation of wall pressure coherence using time-resolved tomographic piv. Experiments in fluids 54:1-15

Schanz D, Gesemann S, and Schröder A (2016) Shake-the-box: Lagrangian particle tracking at high particle image densities. Experiments in fluids 57:1-27

Schneiders JF, Pröbsting S, Dwight RP, van Oudheusden BW, and Scarano F (2016) Pressure estimation from single-snapshot tomographic piv in a turbulent boundary layer. Experiments in Fluids 57:53

Schneiders JF and Scarano F (2016) Dense velocity reconstruction from tomographic ptv with material derivatives. Experiments in fluids 57:1-22

Van Gent P, Michaelis D, Van Oudheusden B, Weiss PÉ, de Kat R, Laskari A, Jeon YJ, David L, Schanz D, Huhn F et al. (2017) Comparative assessment of pressure field reconstructions from particle image velocimetry measurements and lagrangian particle tracking. Experiments in Fluids 58:33

Van Oudheusden B (2013) Piv-based pressure measurement. Measurement Science and Technology 24:032001

Violato D, Moore P, and Scarano F (2011) Lagrangian and eulerian pressure field evaluation of rod-airfoil flow from time-resolved tomographic piv. Experiments in fluids 50:1057-1070 
Wang J, Zhang C, and Katz J (2019) Gpu-based, parallel-line, omni-directional integration of measured pressure gradient field to obtain the 3d pressure distribution. Experiments in Fluids 60:1-24

Wang Z, Gao Q, Wang C, Wei R, and Wang J (2016) An irrotation correction on pressure gradient and orthogonal-path integration for piv-based pressure reconstruction. Experiments in Fluids 57:1-16

Yu H, Kanov K, Perlman E, Graham J, Frederix E, Burns R, Szalay A, Eyink G, and Meneveau C (2012) Studying lagrangian dynamics of turbulence using on-demand fluid particle tracking in a public turbulence database. Journal of Turbulence page N12

Zaman K and Hussain A (1981) Taylor hypothesis and large-scale coherent structures. Journal of Fluid Mechanics 112:379-396 